\title{
Public Key Broadcast Encryption with Low Number of Keys and Constant Decryption Time*
}

\author{
Yi-Ru Liu and Wen-Guey Tzeng \\ Department of Computer Science \\ National Chiao Tung University \\ Hsinchu, Taiwan 30050 \\ wgtzeng@cs.nctu.edu.tw
}

\begin{abstract}
In this paper we propose three public key BE schemes that have efficient complexity measures. The first scheme, called the BE-PI scheme, has $O(r)$ header size, $O(1)$ public keys and $O(\log N)$ private keys per user, where $r$ is the number of revoked users. This is the first public key BE scheme that has both public and private keys under $O(\log N)$ while the header size is $O(r)$. These complexity measures match those of efficient secret key BE schemes.

Our second scheme, called the PK-SD-PI scheme, has $O(r)$ header size, $O(1)$ public key and $O\left(\log ^{2} N\right)$ private keys per user. They are the same as those of the SD scheme. Nevertheless, the decryption time is remarkably $O(1)$. This is the first public key BE scheme that has $O(1)$ decryption time while other complexity measures are kept low. The third scheme, called, the PK-LSD-PI scheme, is constructed in the same way, but based on the LSD method. It has $O(r / \epsilon)$ ciphertext size and $O\left(\log ^{1+\epsilon} N\right)$ private keys per user, where $0<\epsilon<1$. The decryption time is also $O(1)$.

Our basic schemes are one-way secure against full collusion of revoked users in the random oracle model under the BDH assumption. We can modify our schemes to have indistinguishably security against adaptive chosen ciphertext attacks.
\end{abstract}

Keywords: Broadcast encryption, polynomial interpolation, collusion.

\section{Introduction}

Assume that there is a set $\mathcal{U}$ of $N$ users. We would like to broadcast a message to a subset $S$ of them such that only the (authorized) users in $S$ can obtain the message, while the (revoked) users not in $S$ cannot get information about the message. Broadcast encryption is a bandwidth-saving method to achieve this goal via cryptographic key-controlled access. In broadcast encryption, a dealer sets up the system and assigns each user a set of private keys such that the

\footnotetext{
* Research supported in part by NSC projects 96-2628-E-009-011-MY3, 96-3114-P001-002-Y (iCAST), and 96-2219-E-009-013 (TWISC).
} 
broadcasted messages can be decrypted by authorized users only. Broadcast encryption has many applications, such as pay-TV systems, encrypted file sharing systems, digital right management, content protection of recordable data, etc.

A broadcasted message $M$ is sent in the form $\left\langle H d r(S, m), E_{m}(M)\right\rangle$, where $m$ is a session key for encrypting $M$ via a symmetric encryption method $E$. An authorized user in $S$ can use his private keys to decrypt the session key $m$ from $H d r(S, m)$. Since the size of $E_{m}(M)$ is pretty much the same for all broadcast encryption schemes, we are concerned about the header size. The performance measures of a broadcast encryption scheme are the header size, the number of private keys held by each user, the size of public parameters of the system (public keys), the time for encrypting a message, and the time for decrypting the header by an authorized user. A broadcast encryption scheme should be able to resist the collusion attack from revoked users. A scheme is fully collusion-resistant if even all revoked users collude, they get no information about the broadcasted message.

Broadcast encryption schemes can be stateless or stateful. For a stateful broadcast encryption scheme, the private keys of a user can be updated from time to time, while the private keys of a user in a stateless broadcast encryption scheme remain the same through the lifetime of the system. Broadcast encryption schemes can also be public key or secret key. For a public key BE scheme, any one (broadcaster) can broadcast a message to an arbitrary group of authorized users by using the public parameters of the system, while for a secret key broadcast encryption scheme, only the special dealer, who knows the system secrets, can broadcast a message.

In this paper we refer "stateless public key broadcast encryption" as "public key BE".

\subsection{Our Contribution}

We propose three public key BE schemes that have efficient complexity measures. The first scheme, called the BE-PI scheme (broadcast encryption with polynomial interpolation), has $O(r)$ header size, $O(1)$ public keys, and $O(\log N)$ private keys per usen 1 , where $r$ is the number of revoked users. This is the first public key BE scheme that has both public and private keys under $O(\log N)$ while the header size is $O(r)$. These complexity measures match those of efficient secret key BE schemes [112021]. The idea is to run $\log N$ copies of the basic scheme in [171922 in parallel for lifting the restriction on a priori fixed number of revoked users. Nevertheless, if we implement the $\log N$ copies straightforwardly, we would get a scheme of $O(N)$ public keys. We are able to use the properties of bilinear maps as well as special private key assignment to eliminate the need of $O(N)$ public keys and make it a constant number.

Our second scheme, called the PK-SD-PI scheme (public key SD broadcast encryption with polynomial interpolation), is constructed by combining the polynomial interpolation technique and the subset cover method in the SD scheme [16].

\footnotetext{
${ }^{1} \log$ is based on 2 if the base is not specified.
} 
Table 1. Comparison of some fully collusion-resistant public key BE schemes

\begin{tabular}{|c|c|c|c|c|}
\hline & header size & public-key size & private-key size & decryption cost $^{\natural}$ \\
\hline $\mathrm{PK}$ SD-HIBE ${ }^{\dagger}$ & $O(r)$ & $O(1)$ & $O\left(\log ^{2} N\right)$ & $O(\log N)$ \\
\hline BGW-I 4] & $O(1)$ & $O(N)^{b}$ & $O(1)$ & $O(N-r)$ \\
\hline BGW-II 4 & $O(\sqrt{N})$ & $O(\sqrt{N})^{b}$ & $O(1)$ & $O(\sqrt{N})$ \\
\hline BW 5 & $O(\sqrt{N})$ & $O(\sqrt{N})^{b}$ & $O(\sqrt{N})$ & $O(\sqrt{N})$ \\
\hline $\mathrm{LHL}^{\S} 15$ & $O(r D)$ & $O(2 C)^{b}$ & $O(D)$ & $O(C)$ \\
\hline $\mathrm{P}-\mathrm{NP}, \mathrm{P}-\mathrm{TT}, \mathrm{P}-\mathrm{YF}^{\ddagger}$ & $O(r)$ & $O(N)$ & $O(\log N)$ & $O(r)$ \\
\hline Our work: BE-PI & $O(r)$ & $O(1)$ & $O(\log N)$ & $O(r)$ \\
\hline Our work: PK-SD-PI & $O(r)$ & $O(1)$ & $O\left(\log ^{2} N\right)$ & $O(1)$ \\
\hline Our work: PK-LSD-PI & $O(r / \epsilon)$ & $O(1)$ & $O\left(\log ^{1+\epsilon} N\right)$ & $O(1)$ \\
\hline
\end{tabular}

$N$ - the number of users.

$r$ - the number of revoked users.

$\dagger$ - the transformed SD scheme [6] instantiated with constant-size HIBE [2].

$\ddagger$ - the parallel extension of $[17[19] 22$.

b - the public keys are needed for decrypting the header by a user.

$\S-N=C^{D}$.

घ - group operation/modular exponentiation and excluding the time for scanning the header.

The PK-SD-PI scheme has $O(r)$ header size, $O(1)$ public key and $O\left(\log ^{2} N\right)$ private keys per user. They are the same as those of the SD scheme. Nevertheless, the decryption time is remarkably $O(1)$. This is the first public key broadcast encryption scheme that has $O(1)$ decryption time while other complexity measures are kept low. The third scheme, called the PK-LSD-PI scheme, is constructed in the same way, but based on the LSD method. It has $O(r / \epsilon)$ ciphertext size and $O\left(\log ^{1+\epsilon} N\right)$ private keys per user, where $0<\epsilon<1$. The decryption time is also $O(1)$.

Our basic schemes are one-way secure against full collusion of revoked users in the random oracle model under the BDH assumption. We modify our schemes to have indistinguishably security against adaptive chosen ciphertext attacks. The comparison with some other public key BE schemes with full collusion resistance is shown in Table 1 .

\subsection{Related Work}

Fiat and Naor [8] formally proposed the concept of static secret key broadcast encryption. Many researchers followed to propose various broadcast encryption schemes, e.g., see 1112161720.

Kurosawa and Desmedt 13 . proposed a pubic-key BE scheme that is based on polynomial interpolation and traces at most $k$ traitors. The similar schemes of Noar and Pinkas [17, Tzeng and Tzeng [19, and Yoshida and Fujiwara [22] allow revocation of up to $k$ users. Kurosawa and Yoshida [14 generalized the 
polynomial interpolation (in fact, the Reed-Solomon code) to any linear code for constructing public key BE schemes. The schemes in [7/13/14/17/19|22 all have $O(k)$ public keys, $O(1)$ private keys, and $O(r)$ header size, $r \leq k$. However, $k$ is a-priori fixed during the system setting and the public key size depends on it. These schemes can withstand the collusion attack of up to $k$ revoked users only. They are not fully collusion-resistant.

Yoo, et al. 21 observed that the restriction of a pre-fixed $k$ can be lifted by running $\log N$ copies of the basic scheme with different degrees (from $2^{0}$ to $N$ ) of polynomials. They proposed a scheme of $O(\log N)$ private keys and $O(r)$ header size such that $r$ is not restricted. However, their scheme is secret key and the system has $O(N)$ secret values. In the public key setting, the public key size is $O(N)$.

Recently Boneh, et al. 4 proposed a public key BE scheme that has $O(1)$ header size, $O(1)$ private keys, and $O(N)$ public keys. By trading off the header size and public keys, they gave another scheme with $O(\sqrt{N})$ header size, $O(1)$ private keys and $O(\sqrt{N})$ public keys. Lee, et al. 15] proposed a better trade-off by using receiver identifiers in the scheme. It achieves $O(1)$ public key, $O(\log N)$ private keys, but, $O(r \log N)$ header size. Boneh and Waters [5 proposed a scheme that has the traitor tracing capability. This type of schemes $4 \mid 4515$ has the disadvantage that the public keys are needed by a user in decrypting the header. Thus, the de-facto private key of a user is the combination of the public key and his private key.

It is possible to transform a secret key BE scheme into a public key one. For example, Dodis and Fazio [6] transformed the SD and LSD schemes [12]16] into public key SD and LSD schemes, shorted as PK-SD and PK-LSD. The transformation employs the technique of hierarchical identity-based encryption to substitute for the hash function. Instantiated with the newest constant-size hierarchical identity-based encryption 2, the PK-SD scheme has $O(r)$ header size, $O(1)$ public keys and $O\left(\log ^{2} N\right)$ private keys. The PK-LSD scheme has $O(r / \epsilon)$ header size, $O(1)$ public keys and $O\left(\log ^{1+\epsilon} N\right)$ private keys, where $0<$ $\epsilon<1$ is a constant. The decryption costs of the PK-SD and PK-LSD schemes are both $O(\log N)$, which is the time for key derivation incurred by the original relation of private keys. If we apply the HIBE technique to the secret key BE schemes of $O(\log N)$ or $O(1)$ private keys [1|11/20, we would get their public key versions with $O(N)$ private keys and $O(N)$ decryption time.

\section{Preliminaries}

Bilinear map. We use the properties of bilinear maps. Let $G$ and $G_{1}$ be two (multiplicative) cyclic groups of prime order $q$ and $\hat{e}$ be a bilinear map from $G \times G$ to $G_{1}$. Then, $\hat{e}$ has the following properties.

1. For all $u, v \in G$ and $x, y \in Z_{q}, \hat{e}\left(u^{x}, v^{y}\right)=\hat{e}(u, v)^{x y}$.

2. Let $g$ be a generator of $G, \hat{e}(g, g)=g_{1} \neq 1$ is a generator of $G_{1}$. 
BDH hardness assumption. The BDH problem is to compute $\hat{e}(g, g)^{a b c}$ from given $\left(g, g^{a}, g^{b}, g^{c}\right)$. We say that BDH is $(t, \epsilon)$-hard if for any probabilistic algorithm $A$ with time bound $t$, there is some $k_{0}$ such that for any $k \geq k_{0}$,

$$
\operatorname{Pr}\left[A\left(g, g^{a}, g^{b}, g^{c}\right)=\hat{e}(g, g)^{a b c}: g \stackrel{u}{\leftarrow} G ; a, b, c \stackrel{u}{\leftarrow} Z_{q}\right] \leq \epsilon .
$$

Broadcast encryption. A public key BE scheme $\Pi$ consists of three probabilistic polynomial-time algorithms:

- $\operatorname{Setup}\left(1^{z}\right.$, ID, $\left.\mathcal{U}\right)$. Wlog, let $\mathcal{U}=\left\{U_{1}, U_{2}, \ldots, U_{N}\right\}$. It takes as input the security parameter $z$, a system identity ID and a set $\mathcal{U}$ of users and outputs a public key $P K$ and $N$ private key sets $S K_{1}, S K_{2}, \ldots, S K_{N}$, one for each user in $\mathcal{U}$.

- $\operatorname{Enc}(P K, S, M)$. It takes as input the public key PK, a set $S \subseteq \mathcal{U}$ of authorized users and a message $M$ and outputs a pair $\langle H d r(S, m), C\rangle$ of the ciphertext header and body, where $m$ is a randomly generated session key and $C$ is the ciphertext of $M$ encrypted by $m$ via some standard symmetric encryption scheme, e.g., AES.

- $\operatorname{Dec}\left(S K_{k}, H d r(S, m), C\right)$. It takes as input the private key $S K_{k}$ of user $U_{k}$, the header $\operatorname{Hdr}(S, m)$ and the body $C$. If $U_{k} \in S$, it computes the session key $m$ and then uses $m$ to decrypt $C$ for the message $M$. If $U_{k} \notin S$, it cannot decrypt the ciphertext.

The system is correct if all users in $S$ can get the broadcasted message $M$.

Security. We describe the indistinguishability security against adaptive chosen ciphertext attacks (IND-CCA security) for broadcast encryption as follows [4]. Here, we focus on the security of the session key, which in turn guarantees the security of the ciphertext body $C$. Let $E n c^{*}$ and $D e c^{*}$ be like $E n c$ and $D e c$ except that the message $M$ and the ciphertext body $C$ are omitted. The security is defined by an adversary $\mathcal{A}$ and a challenger $\mathcal{C}$ via the following game.

Init. The adversary $\mathcal{A}$ chooses a system identity ID and a target set $S^{*} \subseteq \mathcal{U}$ of users to attack.

Setup. The challenger $\mathcal{C}$ runs $\operatorname{Setup}\left(1^{z}, \mathrm{ID}, \mathcal{U}\right)$ to generate a public key $P K$ and private key sets $S K_{1}, S K_{2}, \ldots, S K_{N}$. The challenger $\mathcal{C}$ gives $S K_{i}$ to $\mathcal{A}$, where $U_{i} \notin S^{*}$.

Query phase 1 . The adversary $\mathcal{A}$ issues decryption queries $Q_{i}, 1 \leq i \leq n$, of form $\left(U_{k}, S, H d r(S, m)\right), S \subseteq S^{*}, U_{k} \in S$, and the challenger $\mathcal{C}$ responds with $\operatorname{Dec}^{*}\left(S K_{k}, H d r(S, m)\right)$, which is the session key encrypted in $\operatorname{Hdr}(S, m)$.

Challenge. The challenger $\mathcal{C}$ runs $E n c^{*}\left(P K, S^{*}\right)$ and outputs $y=H d r\left(S^{*}, m\right)$, where $m$ is randomly chosen. Then, $\mathcal{C}$ chooses a random bit $b$ and a random session key $m^{*}$ and sets $m_{b}=m$ and $m_{1-b}=m^{*}$. $\mathcal{C}$ gives $\left(m_{0}, m_{1}, H d r\left(S^{*}, m\right)\right)$ to $\mathcal{A}$.

Query phase 2. The adversary $\mathcal{A}$ issues more decryption queries $Q_{i}, n+1 \leq$ $i \leq q_{D}$, of form $\left(U_{k}, S, y^{\prime}\right), S \subseteq S^{*}, U_{k} \in S, y^{\prime} \neq y$, and the challenger $\mathcal{C}$ responds with $\operatorname{Dec}^{*}\left(S K_{k}, y^{\prime}\right)$.

Guess. $\mathcal{A}$ outputs a guess $b^{\prime}$ for $b$. 
In the above the adversary A is static since it chooses the target set $S^{*}$ of users before the system setup. Let $\operatorname{Adv}_{\mathcal{A}, I}^{\text {ind-cca }}(z)$ be the advantage that $\mathcal{A}$ wins the above game, that is,

$$
\begin{aligned}
& \operatorname{Adv}_{\mathcal{A}, \Pi}^{\text {ind-cca }}(z)=2 \cdot \operatorname{Pr}\left[\mathcal{A}^{\mathcal{O}}\left(P K, S K_{\mathcal{U} \backslash S^{*}}, m_{0}, m_{1}, \operatorname{Hdr}\left(S^{*}, m\right)\right)=b:\right. \\
& \quad S^{*} \subseteq \mathcal{U},\left(P K, S K_{\mathcal{U}}\right) \leftarrow \operatorname{Setup}\left(1^{z}, \operatorname{ID}, \mathcal{U}\right), \\
& \left.\quad H d r\left(S^{*}, m\right) \leftarrow E n c^{*}\left(P K, S^{*}\right), b \stackrel{u}{\leftarrow}\{0,1\}\right]-1,
\end{aligned}
$$

where $S K_{\mathcal{U}}=\left\{S K_{i}: 1 \leq i \leq N\right\}$ and $S K_{\mathcal{U} \backslash S^{*}}=\left\{S K_{i}: U_{i} \notin S^{*}\right\}$.

Definition 1. A public key BE scheme $\Pi=($ Setup, Enc, Dec $)$ is $\left(t, \epsilon, q_{D}\right)-I N D$ $C C A$ secure if for all $t$-time bounded adversary $\mathcal{A}$ that makes at most $q_{D}$ decryption queries, we have $A d v_{\mathcal{A}, \Pi}^{\text {ind-cca }}(z)<\epsilon$.

In this paper we first give schemes with one-way security against chosen plaintext attacks (OW-CPA security) and then transform them to have IND-CCA security via the Fujisaki-Okamoto transformation 9]. The OW-CPA security is defined as follows.

Init. The adversary $\mathcal{A}$ chooses a system identity ID and a target set $S^{*} \subseteq \mathcal{U}$ of users to attack.

Setup. The challenger $\mathcal{C}$ runs $\operatorname{Setup}\left(1^{z}, \mathrm{ID}, \mathcal{U}\right)$ to generate a public key $P K$ and private key sets $S K_{1}, S K_{2}, \ldots, S K_{N}$. The challenger $\mathcal{C}$ gives $S K_{i}$ to $\mathcal{A}$, where $U_{i} \notin S^{*}$.

Challenge. The challenger $\mathcal{C}$ runs $\operatorname{Enc} c^{*}\left(P K, S^{*}\right)$ and outputs $\operatorname{Hdr}\left(S^{*}, m\right)$, where $m$ is randomly chosen.

Guess. $\mathcal{A}$ outputs a guess $m^{\prime}$ for $m$.

Since $\mathcal{A}$ can always encrypt a chosen plaintext by himself, the oracle of encrypting a chosen plaintext does not matter in the definition. Let $\operatorname{Adv}_{\mathcal{A}, \Pi}^{o w-c p a}(z)$ be the advantage that $\mathcal{A}$ wins the above game, that is,

$$
\begin{aligned}
\operatorname{Adv}_{\mathcal{A}, \Pi}^{\text {ow-cpa }}(z)=\operatorname{Pr}\left[\mathcal{A}\left(P K, S K_{\mathcal{U} \backslash S^{*}}, H d r\left(S^{*}, m\right)\right)\right. & =m: S^{*} \subseteq \mathcal{U}, \\
\left(P K, S K_{\mathcal{U}}\right) \leftarrow \operatorname{Setup}\left(1^{z}, \mathrm{ID}, \mathcal{U}\right), H d r\left(S^{*}, m\right) & \left.\leftarrow \operatorname{Enc}^{*}\left(P K, S^{*}\right)\right] .
\end{aligned}
$$

Definition 2. A public key BE scheme $\Pi=($ Setup, Enc, Dec) is $(t, \epsilon)-O W-C P A$ secure if for all t-time bounded adversary $\mathcal{A}$, we have $\operatorname{Adv}_{\mathcal{A}, \Pi}^{\text {ow-cpa }}(z)<\epsilon$.

\section{The BE-PI Scheme}

Let $G$ and $G_{1}$ be the bilinear groups with the pairing function $\hat{e}$, where $q$ is a large prime. Let $H_{1}, H_{2}:\{0,1\}^{*} \rightarrow G_{1}$ be two hash functions and $E$ be a symmetric encryption with key space $G_{1}$.

The idea of our construction is as follows. For a polynomial $f(x)$ of degree $t$, we assign each user $U_{i}$ a share $f(i)$. The secret is $f(0)$. We can compute the secret $f(0)$ from any $t+1$ shares. If we want to revoke $t$ users, we broadcast their 
shares. Any non-revoked user can compute the secret $f(0)$ from his own share and the broadcasted ones, totally $t+1$ shares. On the other hand, any collusion of revoked users cannot compute the secret $f(0)$ since they have $t$ shares only, including the broadcasted ones. If less than $t$ users are revoked, we broadcast the shares of some dummy users such that $t$ shares are broadcasted totally. In order to achieve $O(r)$ ciphertexts, we use $\log N$ polynomials, each for a range of the number of revoked users.

1. $\operatorname{Setup}\left(1^{z}, \operatorname{ID}, \mathcal{U}\right): z$ is the security parameter, ID is the identity name of the system, and $\mathcal{U}=\left\{U_{1}, U_{2}, \ldots, U_{N}\right\}$ is the set of users in the system. Wlog, let $N$ be a power of 2 . Then, the system dealer does the following:

- Choose a generator $g$ of group $G$, and let $\lg =\log _{g}$ and $g_{1}=\hat{e}(g, g)$.

- Compute $h_{i}=H_{1}(\mathrm{ID} \| i)$ for $1 \leq i \leq \log N$.

- Compute $g^{a_{j}^{(i)}}=H_{2}(\mathrm{ID}\|i\| j)$ for $0 \leq i \leq \log N$ and $0 \leq j \leq 2^{i}$. $\underline{\text { Remark. }}$. The underlying polynomials are, $0 \leq i \leq \log N$,

$$
f_{i}(x)=\sum_{j=0}^{2^{i}} a_{j}^{(i)} x^{j} \quad(\bmod q) .
$$

The system dealer does not know the coefficients $a_{j}^{(i)}=\lg H_{2}(\operatorname{ID}\|i\| j)$. But, this does not matter.

- Randomly choose a secret $\rho \in Z_{q}$ and compute $g^{\rho}$.

- Publish the public key $P K=\left(\mathrm{ID}, H_{1}, H_{2}, E, G, G_{1}, \hat{e}, g, g^{\rho}\right)$.

- Assign a set $S K_{k}=\left\{s_{k, 0}, s_{k, 1}, \ldots, s_{k, \log N}\right\}$ of private keys to user $U_{k}$, $1 \leq k \leq N$, where

$$
s_{k, i}=\left(g^{r_{k, i}}, g^{r_{k, i} f_{i}(k)}, g^{r_{k, i} f_{i}(0)} h_{i}^{\rho}\right)
$$

and $r_{k, i}$ is randomly chosen from $Z_{q}, 1 \leq i \leq \log N$.

2. $\operatorname{Enc}(P K, S, M): S \subseteq \mathcal{U}, R=\mathcal{U} \backslash S=\left\{U_{i_{1}}, U_{i_{2}}, \ldots, U_{i_{l}}\right\}$ is the set of revoked users, where $l \geq 1$. $M$ is the sent message. The broadcaster does the following:

- Let $\alpha=\lceil\log l\rceil$ and $L=2^{\alpha}$.

- Compute $h_{\alpha}=H_{1}(\mathrm{ID} \| \alpha)$.

- Randomly select distinct $i_{l+1}, i_{l+2}, \ldots, i_{L}>N$. These $U_{i_{t}}, l+1 \leq t \leq L$, are dummy users.

- Randomly select a session key $m \in G_{1}$.

- Randomly select $r \in Z_{q}$ and compute, $1 \leq t \leq L$,

$$
g^{r f_{\alpha}\left(i_{t}\right)}=\left(\prod_{j=0}^{L} H_{2}(\mathrm{ID}\|\alpha\| j)^{i_{t}^{j}}\right)^{r} .
$$

- The ciphertext header $H d r(S, m)$ is

$$
\left(\alpha, m \hat{e}\left(g^{\rho}, h_{\alpha}\right)^{r}, g^{r},\left(i_{1}, g^{r f_{\alpha}\left(i_{1}\right)}\right),\left(i_{2}, g^{r f_{\alpha}\left(i_{2}\right)}\right), \ldots,\left(i_{L}, g^{r f_{\alpha}\left(i_{L}\right)}\right)\right) .
$$

- The ciphertext body is $C=E_{m}(M)$. 
3. $\operatorname{Dec}\left(S K_{k}, H d r(S, m), C\right): U_{k} \in S$. The user $U_{k}$ does the following.

- Compute $b_{0}=\hat{e}\left(g^{r}, g^{r_{k, \alpha} f_{\alpha}(k)}\right)=g_{1}^{r r_{k, \alpha} f_{\alpha}(k)}$.

- Compute $b_{j}=\hat{e}\left(g^{r_{k, \alpha}}, g^{r f_{\alpha}\left(i_{j}\right)}\right)=g_{1}^{r r_{k, \alpha} f_{\alpha}\left(i_{j}\right)}, 1 \leq j \leq L$.

- Use the Lagrange interpolation method to compute

$$
g_{1}^{r r_{k, \alpha} f_{\alpha}(0)}=\prod_{j=0}^{L} b_{j}^{\lambda_{j}}
$$

where $\lambda_{j}=\frac{\left(-i_{0}\right)\left(-i_{1}\right) \cdots\left(-i_{j-1}\right)\left(-i_{j+1}\right) \cdots\left(-i_{L}\right)}{\left(i_{j}-i_{0}\right)\left(i_{j}-i_{1}\right) \cdots\left(i_{j}-i_{j-1}\right)\left(i_{j}-i_{j+1}\right) \cdots\left(i_{j}-i_{L}\right)}(\bmod q), i_{0}=k$.

- Compute the session key

$$
\frac{m \hat{e}\left(g^{\rho}, h_{\alpha}\right)^{r} \cdot g_{1}^{r r_{k, \alpha} f_{\alpha}(0)}}{\hat{e}\left(g^{r}, g^{r_{k, \alpha} f_{\alpha}(0)} h_{\alpha}^{\rho}\right)}=\frac{m \hat{e}\left(g^{\rho}, h_{\alpha}\right)^{r} \cdot g_{1}^{r r_{k, \alpha} f_{\alpha}(0)}}{\hat{e}\left(g^{r}, h_{\alpha}^{\rho}\right) \cdot g_{1}^{r r_{k, \alpha} f_{\alpha}(0)}}=m .
$$

- Use $m$ to decrypt the ciphertext body $C$ to obtain the message $M$.

Correctness. We can easily see that the scheme is correct by Equation (2).

\subsection{Performance Analysis}

For each system, the public key is (ID, $H_{1}, H_{2}, E, G, G_{1}, \hat{e}, g, g^{\rho}$ ), which is of size $O(1)$. Since all systems can use the same $\left(H, E, G, G_{1}, \hat{e}, g\right)$, the public key specific to a system is simply (ID, $\left.g^{\rho}\right)$. Each system dealer has a secret $\rho$ for assigning private keys to its users. Each user $U_{k}$ holds private keys $S K_{k}=$ $\left\{s_{k, 0}, s_{k, 1}, \ldots, s_{k, \log N}\right\}$, each corresponding to a share of polynomial $f_{i}$ in the masked form, $0 \leq i \leq \log N$. The number of private keys is $O(\log N)$. When $r$ users are revoked, we choose the polynomial $f_{\alpha}$ of degree $2^{\alpha}$ for encrypting the session key, where $2^{\alpha-1}<r \leq 2^{\alpha}$. Thus, the header size is $O\left(2^{\alpha}\right)=O(r)$. It is actually no more than $2 r$.

To prepare a header, the broadcaster needs to compute one pairing function, $2^{\alpha}+2$ hash functions, and $2^{\alpha}+2$ modular exponentiations, which is $O(r)$ modular exponentiations.

For a user in $S$ to decrypt a header, with a little re-arrangement of Equation (11) as

$$
\prod_{j=0}^{L} b_{j}^{\lambda_{j}}=b_{0}^{\lambda_{0}} \cdot \hat{e}\left(g^{r_{k, \alpha}}, \prod_{j=1}^{L}\left(g^{r f_{\alpha}\left(i_{j}\right)}\right)^{\lambda_{j}}\right),
$$

the user needs to perform 3 pairing functions and $2^{\alpha}$ modular exponentiations, which is $O(r)$ modular exponentiations. The evaluation of $\lambda_{j}$ 's can be done in $O(L)=O(2 r)$ if the header consists of

$$
\tilde{\lambda}_{j}=\frac{\left(-i_{1}\right) \cdots\left(-i_{j-1}\right)\left(-i_{j+1}\right) \cdots\left(-i_{L}\right)}{\left(i_{j}-i_{1}\right) \cdots\left(i_{j}-i_{j-1}\right)\left(i_{j}-i_{j+1}\right) \cdots\left(i_{j}-i_{L}\right)} \bmod q, 1 \leq j \leq L .
$$

The user can easily compute $\lambda_{j}$ 's from $\tilde{\lambda}_{j}$ 's. Inclusion of $\tilde{\lambda}_{j}$ 's in the header does not affect the order of the header size. 


\subsection{Security Analysis}

We show that it has OW-CPA security in the random oracle model under the BDH assumption.

Theorem 1. Assume that the BDH problem is $\left(t_{1}, \epsilon_{1}\right)$-hard. Our BE-PI scheme is $\left(t_{1}-t^{\prime}, \epsilon_{1}\right)$-OW-CPA secure in the random oracle model, where $t^{\prime}$ is some polynomially bounded time.

Proof. We reduce the BDH problem to the problem of computing the session key from the header by the revoked users. Since the polynomials $f_{i}(x)=\sum_{j=0}^{L} a_{j}^{(i)} x^{j}$ and secret shares of users for the polynomials are independent for different $i$ 's, we simply discuss security for a particular $\alpha$. Wlog, let $R=\left\{U_{1}, U_{2}, \ldots, U_{L}\right\}$ be the set of revoked users and the target set of attack be $S^{*}=\mathcal{U} \backslash R$. Note that $S^{*}$ was chosen by the adversary in the Init stage. Let the input of the BDH problem be $\left(g, g^{a}, g^{b}, g^{c}\right)$, where the pairing function is implicitly known. We set the system parameters as follows:

1. Randomly select $\tau, \kappa, \mu_{1}, \mu_{2}, \ldots, \mu_{L}, w_{1}, w_{2}, \ldots, w_{L} \in Z_{q}$.

2. Set the public key of the system:

(a) Let the input $g$ be the generator $g$ in the system.

(b) Set $g^{\rho}=g^{a}$.

(c) The public key is (ID, $H_{1}, H_{2}, E, G, G_{1}, \hat{e}, g, g^{a}$ ).

(d) The following is implicitly computed.

- Set $f_{\alpha}(i)=w_{i}, 1 \leq i \leq L$.

- Let $g^{a_{0}^{(\alpha)}}=g^{f_{\alpha}(0)}=g^{a} \cdot g^{\tau}=g^{a+\tau}$.

- Compute $g^{a_{i}^{(\alpha)}}, 1 \leq i \leq L$, from $g^{a_{0}^{(\alpha)}}$ and $g^{f_{\alpha}(j)}=g^{w_{j}}, 1 \leq j \leq L$, by the Lagrange interpolation method over exponents.

- Set $h_{\alpha}=g^{b} \cdot g^{\kappa}=g^{b+\kappa}$.

- For $j \neq \alpha$, choose a random polynomial $f_{j}(x)$ and set $h_{j}=g^{z_{j}}$, where $z_{j}$ is randomly chosen from $Z_{q}$.

3. Set the secret keys $\left(g^{r_{i, j}}, g^{r_{i, j} f_{j}(i)}, g^{r_{i, j} f_{j}(0)} h_{j}^{\rho}\right), 0 \leq j \leq \log N$, of the revoked user $U_{i}, 1 \leq i \leq L$, as follows:

(a) For $j=\alpha$, let $g^{r_{i, \alpha}}=g^{-b+\mu_{i}}, g^{r_{i, \alpha} f_{\alpha}(i)}=\left(g^{r_{i, \alpha}}\right)^{w_{i}}$, and $g^{r_{i, \alpha} f_{\alpha}(0)} h_{\alpha}^{\rho}=g^{\left(-b+\mu_{i}\right)(a+\tau)}\left(g^{b+\kappa}\right)^{a}=g^{a\left(\mu_{i}+\kappa\right)-b \tau+\mu_{i} \tau}$.

(b) For $j \neq \alpha$, randomly choose $r_{i, j} \in Z_{q}$ and compute $g^{r_{i, j}}, g^{r_{i, j} f_{j}(i)}$ and $g^{r_{i, j} f_{j}(0)} h_{j}^{\rho}=g^{r_{i, j} f_{j}(0)}\left(g^{a}\right)^{z_{j}}$.

4. Set the header $\left(\alpha, m \hat{e}\left(g^{\rho}, h_{\alpha}\right)^{r}, g^{r},\left(1, g^{r f_{\alpha}(1)}\right),\left(2, g^{r f_{\alpha}(2)}\right), \ldots,\left(L, g^{r f_{\alpha}(L)}\right)\right)$ as follows:

(a) Let $g^{r}=g^{c}$.

(b) Compute $g^{r f_{\alpha}(i)}=\left(g^{c}\right)^{w_{i}}, 1 \leq i \leq L$.

(c) Randomly select $y \in G_{1}$ and set $m \hat{e}\left(g^{\rho}, h_{\alpha}\right)^{r}=y$. We do not know what $m$ is. But, this does not matter.

Assume that the revoked users together can compute the session key $m$. During computation, the users can query $H_{1}$ and $H_{2}$ hash oracles. If the query is of the form $H_{2}(\mathrm{ID}\|i\| j)$ or $H_{1}(\mathrm{ID} \| i)$, we set them to be $g^{a_{j}^{(i)}}$ and $h_{i}$, respectively. 
If the query has ever been asked, we return the stored hash value for the query. For other non-queried inputs, we return random values in $G$.

We should check whether the distributions of the parameters in our reduction and those in the system are equal. We only check those related to $\alpha$ since the others are correctly distributed. Since $\tau, w_{1}, w_{2}, \ldots, w_{L}$ are randomly chosen, $g^{a_{i}^{(\alpha)}}, 0 \leq i \leq L$ are uniformly distributed over $G^{L+1}$. Due to the random oracle model, their corresponding system parameters are also uniformly distributed over $G^{L+1}$. Since $\kappa, \mu_{1}, \mu_{2}, \ldots, \mu_{L}$ are randomly chosen, the distribution of $h_{\alpha}$ and $g^{r_{i, \alpha}}, 1 \leq i \leq L$, are uniform over $G^{L+1}$, which is again the same as that of the corresponding system parameters. The distributions of $g^{r}$ in the header and $g^{\rho}$ in the public key are both uniform over $G$ since they are set from the given input $g^{c}$ and $g^{a}$, respectively. Since the session key $m$ is chosen randomly from $G_{1}, m \hat{e}\left(g^{\rho}, h_{\alpha}\right)^{r}$ is distributed uniformly over $G_{1}$. We set it to a random value $y \in G_{1}$. Even though we don't know about $m$, it does not affect the reduction. Other parameters are dependent on what have been discussed. We can check that they are all computed correctly. So, the reduction preserves the right distribution.

If the revoked users compute $m$ from the header with probability $\epsilon$, we can solve the $\mathrm{BDH}$ problem with the same probability $\epsilon_{1}=\epsilon$ by computing the following:

$$
\begin{aligned}
y \cdot m^{-1} \cdot \hat{e}\left(g^{a}, g^{c}\right)^{-\kappa} & =\hat{e}\left(g^{\rho}, h_{\alpha}\right)^{r} \cdot \hat{e}(g, g)^{-a c \kappa} \\
& =\hat{e}\left(g^{a}, g^{b+\kappa}\right)^{c} \cdot \hat{e}(g, g)^{-a c \kappa} \\
& =\hat{e}(g, g)^{a b c}
\end{aligned}
$$

Let $t^{\prime}$ be the time for this reduction and the solution computation in Equation (3). We can see that $t^{\prime}$ is polynomially bounded. Thus, if the collusion attack of the revoked users takes $t_{1}-t^{\prime}$ time, we can solve the BDH problem within time $t_{1}$.

\section{The BE-PI Scheme with IND-CCA Security}

In Theorem 1, we show that the session key in the header is one-way secure against any collusion of revoked users. There are some standard techniques of transforming OW-CPA security to IND-CCA security. Here we present such a scheme $\Pi^{\prime}$ based on the technique in 9 .

The IND-CCA security of the Fujisaki-Okamoto transformation depends only on the OW-CPA security of the public key encryption scheme, the FG security of a symmetric encryption scheme $\mathcal{E}$, and the $\gamma$-uniformity of the public key encryption scheme. The FG-security is the counterpart of the IND-security for symmetric encryption. A public key encryption scheme is $\gamma$-uniform if for every key pair $(p k, s k)$, every message $x$, and $y \in\{0,1\}^{*}, \operatorname{Pr}\left[E_{p k}(x)=y\right] \leq \gamma$. Before applying the transformation, we check the following things:

1. The transformation applies to public key encryption, while ours is public key broadcast encryption. Nevertheless, if the authorized set $S$ is fixed, our public 
key broadcast encryption scheme is a public key encryption scheme with public key $p k=(P K, S)$. In the definition of IND-CCA security (Definition $1)$, the adversary $\mathcal{A}$ selects a target set $S^{*}$ of users to attack in the Init stage and $S^{*}$ is fixed through the rest of the attack. Thus, we can discuss the attack of $\mathcal{A}$ with a fixed target set $S^{*}$. Note that $\mathcal{A}$ is a static adversary.

2. Let $S$ be a fixed authorized set of users. For every $m$ and every $y \in\{0,1\}^{*}$, $\operatorname{Pr}[\operatorname{Hdr}(S, m)=y]$ is either 0 or $1 / q \simeq 1 / 2^{z}$, where $z$ is the security parameter (the public key size). Thus, our broadcast encryption scheme is $2^{-z}$-uniform if the authorized set is fixed.

Let $\mathcal{E}: K \times G_{1} \rightarrow G_{1}$ be a symmetric encryption scheme with FG-security, where $K$ is the key space of $\mathcal{E}$. Let $H_{3}: G_{1} \times G_{1} \rightarrow Z_{q}$ and $H_{4}: G_{1} \rightarrow K$ be two hash functions. The modification of $\Pi$ for $\Pi^{\prime}$ is as follows.

- In the Setup algorithm, add $\mathcal{E}, H_{3}, H_{4}$ to PK.

- In the Enc algorithm,

$$
\begin{aligned}
H d r(S, m)= & \left(g^{r}, \sigma \hat{e}\left(g^{\rho}, h_{\alpha}\right)^{r}, \mathcal{E}_{H_{4}(\sigma)}(m),\right. \\
& \left.\left(i_{1}, g^{r f_{\alpha}\left(i_{1}\right)}\right),\left(i_{2}, g^{r f_{\alpha}\left(i_{2}\right)}\right), \ldots,\left(i_{L}, g^{r f_{\alpha}\left(i_{L}\right)}\right)\right),
\end{aligned}
$$

where $\sigma$ is randomly chosen from $G_{1}$ and $r=H_{3}(\sigma, m)$.

- In the Dec algorithm, we first compute $\bar{\sigma}$ as described in the BE-PI scheme. Then, we compute the session key $\bar{m}$ from $\mathcal{E}_{H_{4}(\sigma)}(m)$ by using $\bar{\sigma}$. We check whether $\sigma \hat{e}\left(g^{\rho}, h_{\alpha}\right)^{r}=\bar{\sigma} \hat{e}\left(g^{\rho}, h_{\alpha}\right)^{H_{3}(\bar{\sigma}, \bar{m})}$ and $g^{r f_{\alpha}\left(i_{j}\right)}=g^{f_{\alpha}\left(i_{j}\right) H_{3}(\bar{\sigma}, \bar{m})}, 1 \leq$ $j \leq L$. If they are all equal, $\bar{m}$ is outputted. Otherwise, $\perp$ is outputted.

Let $q_{H_{3}}, q_{H_{4}}$ and $q_{D}$ be the numbers of queries to $H_{3}, H_{4}$ and the decryption oracles, respectively. Our scheme $\Pi^{\prime}$ is IND-CCA-secure.

Theorem 2. Assume that the BDH problem is $\left(t_{1}, \epsilon_{1}\right)$-hard and the symmetric encryption $\mathcal{E}$ is $\left(t_{2}, \epsilon_{2}\right)$ FG-secure. The scheme $\Pi^{\prime}$ is $\left(t, \epsilon, q_{H_{3}}, q_{H_{4}}, q_{D}\right)$-IND$C C A$ secure in the random oracle model, where $t^{\prime}$ is some polynomially bounded time,

$$
\begin{aligned}
& t=\min \left\{t_{1}-t^{\prime}, t_{2}\right\}-O\left(2 z\left(q_{H_{3}}+q_{H_{4}}\right)\right) \text { and } \\
& \epsilon=\left(1+2\left(q_{H_{3}}+q_{H_{4}}\right) \epsilon_{1}+\epsilon_{2}\right)\left(1-2 \epsilon_{1}-2 \epsilon_{2}-2^{-z+1}\right)^{-q_{D}}-1 .
\end{aligned}
$$

This theorem is proved by showing that if $\Pi^{\prime}$ is not IND-CCA-secure, then either $\Pi$ is not OW-CPA-secure or $\mathcal{E}$ is not FG-secure directly. The OW-CPA security of $\Pi$ is based on the $\mathrm{BDH}$ assumption. We note that the application of the transformation to other types of schemes could be delicate. Galindo [10] pointed out such a case. Nevertheless, the problem occurs in the proof and is fixable without changing the transformation or the assumption. The detailed proof will be given in the full version of the paper.

\section{A Public Key SD Scheme}

In the paradigm of subset cover for broadcast encryption [16, the system chooses a collection $\mathcal{C}$ of subsets of users such that each set $S$ of users can be covered by 
the subsets in $\mathcal{C}$, that is, $S=\cup_{i=1}^{w} S_{w}$, where $S_{i} \in \mathcal{C}$ are disjoint, $1 \leq i \leq w$. Each subset $S_{i}$ in $\mathcal{C}$ is associated with a private key $k_{i}$. A user is assigned a set of keys such that he can derive the private keys of the subsets to which he belongs. The subset keys $k_{i}$ cannot be independent. Otherwise, each user may hold too many keys. It is preferable that the subset keys have some relations, for example, one can be derived from another. Thus, each user $U_{k}$ is given a set $S K_{k}$ of keys so that he can derive the private key of a subset to which he belongs. A subset-cover based broadcast encryption scheme plays the art of choosing a collection $\mathcal{C}$ of subsets, assigning subset and user keys, and finding subset covers.

\subsection{The PK-SD-PI Scheme}

We now present our PK-SD-PI scheme, which is constructed by using the polynomial interpolation technique on the collection of subsets in [16. The system setup is similar to that of the BE-PI scheme. Consider a complete binary tree $T$ of $\log N+1$ levels. The nodes in $T$ are numbered differently. Each user in $\mathcal{U}$ is associated with a different leaf node in $T$. We refer to a complete subtree rooted at node $i$ as "subtree $T_{i}$ ". For each subtree $T_{i}$ of $\eta$ levels (level 1 to level $\eta$ from top to bottom), we define the degree-1 polynomials

$$
f_{j}^{(i)}(x)=a_{j, 1}^{(i)} x+a_{j, 0}^{(i)} \quad(\bmod q),
$$

where $a_{j, 0}^{(i)}=\lg H_{2}(\operatorname{ID}\|i\| j \| 0)$ and $a_{j, 1}^{(i)}=\lg H_{2}(\operatorname{ID}\|i\| j \| 1), 2 \leq j \leq \eta$. For a user $U_{k}$ in the subtree $T_{i}$ of $\eta$ levels, he is given the private keys

$$
s_{k, i, j}=\left(g^{r_{k, i, j}}, g^{r_{k, i, j} f_{j}^{(i)}\left(i_{j}\right)}, g^{r_{k, i, j} f_{j}^{(i)}(0)} h^{\rho}\right)
$$

for $2 \leq j \leq \eta$, where nodes $i_{1}, i_{2}, \ldots, i_{\eta}$ are the nodes in the path from node $i$ to the leaf node for $U_{k}$ (including both ends). We can read $s_{k, i, j}$ as the private key of $U_{k}$ for the $j$ th level of subtree $T_{i}$. In Figure 1, the private keys (in the unmasked form) of $U_{1}$ and $U_{3}$ for subtree $T_{i}$ with $\eta=4$ are given. Here, we use $h^{\rho}$ in all private keys in order to save space in the header.

Recall that in the SD scheme, the collection $\mathcal{C}$ of subsets is

$$
\left\{S_{i, t}: \text { node } i \text { is a parent of node } t, i \neq t\right\},
$$

where $S_{i, t}$ denotes the set of users in subtree $T_{i}$, but not in subtree $T_{t}$. By our design, if the header contains a masked share for $f_{j}^{(i)}(t)$, where node $t$ is in the $j$-th level of subtree $T_{i}$, only user $U_{k}$ in $S_{i, t}$ can decrypt the header by using his private key $s_{k, i, j}$, that is, the masked form of $f_{j}^{(i)}(s)$, for some $s \neq t$. In Figure 1, the share $f_{3}^{(i)}(t)$ is broadcasted so that only the users in $S_{i, t}$ can decrypt the header.

For a set $R$ of revoked users, let $S_{i_{1}, t_{1}}, S_{i_{2}, t_{2}}, \ldots, S_{i_{z}, t_{z}}$ be a subset cover for $\mathcal{U} \backslash R$, the header is

$$
\left(m \hat{e}\left(g^{\rho}, h\right)^{r}, g^{r},\left(i_{1}, t_{1}, g^{r f_{j_{1}}^{\left(i_{1}\right)}\left(t_{1}\right)}\right), \ldots,\left(i_{z}, t_{z}, g^{r f_{j_{z}}^{\left(i_{z}\right)}\left(t_{z}\right)}\right)\right),
$$

where node $t_{k}$ is in the $j_{k}$-th level of subtree $T_{i_{k}}, 1 \leq k \leq z$. 


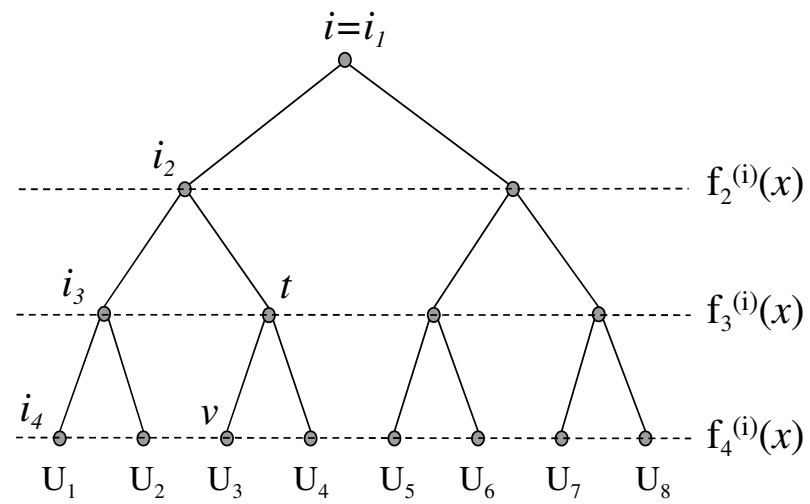

- $\mathrm{U}_{1}$ holds masked shares of $\mathrm{f}_{2}^{(\mathrm{i})}\left(i_{2}\right), \mathrm{f}_{3}^{(\mathrm{i})}\left(i_{3}\right), \mathrm{f}_{4}^{(\mathrm{i})}\left(i_{4}\right)$

$-\mathrm{U}_{3}$ holds masked shares of $\mathrm{f}_{2}{ }^{(\mathrm{i})}\left(i_{2}\right), \mathrm{f}_{3}{ }^{(\mathrm{i})}(t), \mathrm{f}_{4}{ }^{(\mathrm{i})}(v)$

- For subset $\mathrm{S}_{i, t}$, a masked share of $\mathrm{f}_{3}{ }^{(\mathrm{i})}(t)$ is broadcasted so that

$\mathrm{U}_{3}$ and $\mathrm{U}_{4}$ cannot decrypt, but others can.

Fig. 1. Level polynomials, private keys and broadcasted shares for subtree $T_{i}$

For decryption, a non-revoked user finds $i_{k}, t_{k}, g^{r f_{j_{k}}^{\left(i_{k}\right)}\left(t_{k}\right)}$ (corresponding to $S_{i_{k}, t_{k}}$ where he is in) from the header and applies the Lagrange interpolation to compute the session key $m$.

Performance. The public key is $O(1)$, which is the same as that of the BE-PI scheme. Each user belongs to at most $\log N+1$ subtrees and each subtree has at most $\log N+1$ levels. For the subtree of $\eta$ levels, the user in the subtree holds $\eta-1$ private keys. Thus, the total number of shares (private keys) held by each user is $\sum_{i=1}^{\log N} i=O\left(\log ^{2} N\right)$. According to [16], the number $z$ of subsets in a subset cover is at most $2|R|-1$, which is $O(r)$.

When the header streams in, a non-revoked user $U_{k}$ looks for his containing subset $S_{i_{j}, t_{j}}$ to which he belongs. With a proper numbering of the nodes in $T$, this can be done very fast, for example, in $O(\log \log N)$ time. Without considering the time of scanning the header to find out his containing subset, each user needs to perform 2 modular exponentiations and 3 pairing functions. Thus, the decryption cost is $O(1)$.

Security. We first show that the scheme is one-way secure.

Theorem 3. Assume that the BDH problem is $\left(t_{1}, \epsilon_{1}\right)$-hard. Our PK-SD-PI scheme is $\left(t_{1}-t^{\prime}, \epsilon_{1}\right)$-OW-CPA secure in the random oracle model, where $t^{\prime}$ is some polynomially bounded time.

Proof. The one-way security proof for the PK-SD-PI scheme is similar to that for the BE-PI scheme. In the PK-SD-PI scheme, all polynomials $f_{j}^{(i)}(x)$ are of degree one. Let $\left(g, g^{a}, g^{b}, g^{c}\right)$ be the input to the BDH problem. Let $S_{i_{1}, t_{1}}, S_{i_{2}, t_{2}}, \ldots, S_{i_{z}, t_{z}}$ be a subset cover for $S^{*}=\mathcal{U} \backslash R$. Due to the random oracle assumption for $H_{1}$ 
and $H_{2}$, all polynomials are independent. Thus, we can simply consider a particular $S_{\alpha, t}$ in the subset cover for $S^{*}=\mathcal{U} \backslash R$, where $t$ is at level $\beta$ of subtree $T_{\alpha}$. The corresponding polynomial is $f(x)=f_{\beta}^{(\alpha)}(x)=a_{1} x+a_{0}(\bmod q)$. Wlog, let $\left\{U_{1}, U_{2}, \ldots, U_{l}\right\}$ be the set of revoked users that have the secret share about $f(t)$. The reduction to the $\mathrm{BDH}$ problem is as follows. Recall that the public key of the PK-SD-PI method is (ID $H_{1}, H_{2}, E, G, G_{1}, \hat{e}, g, g^{\rho}$ ).

1. Let $g$ be the generator in the system and $g^{\rho}=g^{a}$.

2. Set $f(t)=w$ and compute $g^{f(t)}=g^{w}$, where $w$ is randomly chosen from $Z_{q}$.

3. Let $g^{a_{0}}=g^{f(0)}=g^{a} \cdot g^{\tau}$, where $\tau$ is randomly chosen from $Z_{q}$.

4. Compute $g^{a_{1}}$ from $g^{f(t)}$ and $g^{a_{0}}$ via the Lagrange interpolation.

5. The (random) hash values $H_{2}(\mathrm{ID}\|\alpha\| \beta \| 0)$ and $H_{2}(\mathrm{ID}\|\alpha\| \beta \| 1)$ are set as $g^{a_{0}}$ and $g^{a_{1}}$ respectively.

6. Set $h=g^{b} \cdot g^{\kappa}$, where $\kappa$ is randomly chosen from $Z_{q}$.

7. The $f(x)$-related secret share of $U_{i}, 1 \leq i \leq l$, is computed as $\left(g^{r_{i}}, g^{r_{i} f(t)}\right.$, $\left.g^{r_{i} f(0)} h^{\rho}\right)$, where $g^{r_{i}}=g^{-b} \cdot g^{\mu_{i}}$ and $\mu_{i}$ is randomly chosen from $Z_{q}$. Note that $g^{r_{i} f(0)} h^{\rho}=g^{a\left(\mu_{i}+\kappa\right)-b \tau+\mu_{i} \tau}$ can be computed from the setting in the previous steps.

8. The non- $f(x)$-related secret shares of $U_{i}, 1 \leq i \leq l$, can be set as follows. Let $f^{\prime}$ be a polynomial related to subtree $\alpha^{\prime}$ and level $\beta^{\prime}$, where $t^{\prime}$ is in the $\beta^{\prime}$-th level and $U_{i} \in S_{\alpha^{\prime}, t^{\prime}}$. The secret share $\left(g^{r_{i}^{\prime}}, g^{r_{i}^{\prime} f^{\prime}}\left(t^{\prime}\right), g^{r_{i}^{\prime}} f^{\prime}(0) h^{\rho}\right)$ of $U_{i}$ is computed from $\left(g^{r_{i}}, g^{r_{i} f(t)}, g^{r_{i} f(0)} h^{\rho}\right)$. Let $f^{\prime}\left(t^{\prime}\right)=w^{\prime}, f^{\prime}(0)=f(0)+a^{\prime}$ and $r_{i}^{\prime}=r_{i}+r^{\prime}$, where $w^{\prime}, a^{\prime}$, and $r^{\prime}$ are randomly chosen from $Z_{q}$. Thus, $g^{r_{i}^{\prime}}=$ $g^{r_{i}} \cdot g^{r^{\prime}}, g^{r_{i}^{\prime} f^{\prime}\left(t^{\prime}\right)}=\left(g^{r_{i}^{\prime}}\right) w^{w^{\prime}}$ and $g^{r_{i}^{\prime} f^{\prime}(0)} h^{\rho}=\left(g^{r_{i} f(0)} h^{\rho}\right) \cdot g^{r^{\prime}} f(0) \cdot g^{r_{i} a^{\prime}} \cdot g^{r^{\prime} a^{\prime}}$. Note that the hash values $H_{2}\left(\mathrm{ID}\left\|\alpha^{\prime}\right\| \beta^{\prime} \| 0\right)$ and $H_{2}\left(\mathrm{ID}\left\|\alpha^{\prime}\right\| \beta^{\prime} \| 1\right)$ can be answered accordingly.

9. Set the challenge as

$$
\left(y, g^{c},\left(i_{1}, t_{1}, g^{c f_{j_{1}}^{\left(i_{1}\right)}\left(t_{1}\right)}\right),\left(i_{2}, t_{2}, g^{c f_{j_{2}}^{\left(i_{2}\right)}\left(t_{2}\right)}\right), \ldots,\left(i_{z}, t_{z}, g^{c f_{j_{z}}^{\left(i_{z}\right)}\left(t_{z}\right)}\right)\right),
$$

where $y$ is randomly chosen from $G$ and thought as $m \hat{e}\left(g^{\rho}, h\right)^{c}$. Note that $g^{c f_{j_{k}}^{\left(i_{k}\right)}\left(t_{k}\right)}, 1 \leq k \leq z$, can be computed since $f_{j_{k}}^{\left(i_{k}\right)}\left(t_{k}\right)$ is a number randomly chosen from $Z_{q}$, as described in Step 2.

If the revoked users $U_{1}, U_{2}, \ldots, U_{l}$ can together compute the session key $m$ from the challenge with probability $\epsilon_{1}$, we can compute

$$
\begin{aligned}
y \cdot m^{-1} \cdot \hat{e}\left(g^{a}, g^{c}\right)^{-\kappa} & =\hat{e}\left(g^{\rho}, h\right)^{c} \cdot \hat{e}(g, g)^{-a c \kappa} \\
& =\hat{e}\left(g^{a}, g^{b+\kappa}\right)^{c} \cdot \hat{e}(g, g)^{-a c \kappa}=\hat{e}(g, g)^{a b c}
\end{aligned}
$$

with the same probability $\epsilon_{1}$. This contradicts the BDH assumption.

Let $t^{\prime}$ be the time for the reduction and solution computation in Equation (4), where $t^{\prime}$ is polynomially bounded. Thus, if the collusion attack takes $t_{1}-t^{\prime}$, we can solve the $\mathrm{BDH}$ problem in time $t_{1}$.

Similarly, we can modify our PK-SD-PI scheme to have IND-CCA security like Section 4 


\subsection{The PK-LSD-PI Scheme}

The LSD method is an improvement of the SD method by using a sub-collection $\mathcal{C}^{\prime}$ of $\mathcal{C}$ in the SD method. The basic observation is that $S_{i, t}$ can be decomposed to $S_{i, k} \cup S_{k, t}$. The LSD method delicately selects $C^{\prime}$ such that each $S_{i, t} \in \mathcal{C}$ is either in $\mathcal{C}^{\prime}$ or equal to $S_{i, k} \cup S_{k, t}$, where $S_{i, k}$ and $S_{k, t}$ are in $\mathcal{C}^{\prime}$. The subset cover found for $\mathcal{U} \backslash R$ in the SD method is used except that each $S_{i, t}$ in the cover, but not in $C^{\prime}$, is replaced by two subsets $S_{i, k}$ and $S_{k, t}$ in $C^{\prime}$. Thus, each user belongs to a less number of $S_{i, t}$ 's in $C^{\prime}$ such that it holds a less number of private keys.

We consider the basic case of the LSD method, in which each user holds $(\log n)^{3 / 2}$ private keys. There are $\sqrt{\log n}$ "special" levels in $T$. The root is at a special level and every level of depth $k \cdot \sqrt{\log n}, 1 \leq k \leq \sqrt{\log n}$, is special. A layer is the set of the levels between two adjacent special levels. Each layer has $\sqrt{\log n}$ levels. The collection $\mathcal{C}^{\prime}$ of the LSD method is

$\left\{S_{i, t}:\right.$ nodes $i$ and $t$ are in the same layer, or node $i$ is at a special level $\}$.

There are two types of $S_{i, t}$ 's in $\mathcal{C}^{\prime}$. The first type is that node $i$ is in a special level and the second type is that nodes $i$ and $t$ are in the same layer. Every non-revoked set $\mathcal{U} \backslash R$ can be covered by at most $4|R|-2$ disjoint subsets in $\mathcal{C}^{\prime}$.

Our PK-LSD-PI scheme is as follows. Since $\mathcal{C}^{\prime}$ is just a sub-collection of $\mathcal{C}$ in the SD method, our PK-LSD-PI scheme is almost the same as the PK-SDPI scheme except that some polynomials for type-2 $S_{i, t} \in \mathcal{C}^{\prime}$ are unnecessary. Consider a user $U_{k}$ (or its corresponding leaf node). For his ancestor node $i$ at a special layer (type- $1 S_{i, t}$ 's), $U_{k}$ is given the private keys (corresponding to subtree $T_{i}$ ) by the same way as the PK-SD-PI method. There are $\sqrt{\log n}$ such $i$ 's and each $T_{i}$ has at most $\log n$ levels. In this case, $U_{k}$ holds $(\log n)^{3 / 2}$ private keys. For his ancestor node $i$ and nodes $t$ in the same layer (type-2 $S_{i, t}$ 's), choose degree-1 polynomials for the levels between $i$ and its (underneath) adjacent special level only. There are at most $\sqrt{\log n}$ such polynomials and $U_{k}$ is assigned corresponding $\sqrt{\log n}$ private keys as the PK-SD-PI scheme does. In this case, $U_{k}$ holds at $\operatorname{most} \log n \cdot \sqrt{\log n}$ private keys since $U_{k}$ has $\log n$ ancestors. Overall, each user $U_{k}$ holds at most $2(\log n)^{3 / 2}$ private keys.

Security. We show that the scheme described in this subsection is one-way secure.

Theorem 4. Assume that the BDH problem is $\left(t_{1}, \epsilon_{1}\right)$-hard. Our PK-LSD-PI scheme is $\left(t_{1}-t^{\prime}, \epsilon_{1}\right)-O W$-CPA secure in the random oracle model, where $t^{\prime}$ is some polynomially bounded time.

Proof. The collection of $S_{i, t}$ 's for covering $\mathcal{U} \backslash R$ in the LSD method is a subcollection of that in the SD method. The way of assigning private keys to users is the same as that of the PK-SD-PI scheme except that we omit the polynomials that are never used due to the way of choosing a subset cover in the LSD method. In the random oracle model, we can simply consider a particular $S_{\alpha, t}$ in the subset cover for $\mathcal{U} \backslash R$. Since all conditions are the same, the rest of proof is the same as that in Theorem 3 . 
With the same extension in [12, we can have a PK-LSD-PI scheme that has $O(1)$ public keys and $O\left(\log ^{1+\epsilon}\right)$ private keys, for any constant $0<\epsilon<1$. The header size is $O(r / \epsilon)$, which is $O(r)$ for a constant $\epsilon$. The decryption cost excluding the time of scanning the header is again $O(1)$.

\section{Conclusion}

We have presented very efficient public key BE schemes. They have low public and private keys. Two of them even have a constant decryption time. Our results show that the efficiency of public key BE schemes is comparable to that of private-key BE schemes.

We are interested in reducing the ciphertext size while keeping other complexities low in the future.

\section{Acknowledgement}

We thank Eike Kiltz and Michel Abdalla for valuable comments on the manuscript.

\section{References}

1. Attrapadung, N., Imai, H.: Graph-decomposition-based frameworks for subsetcover broadcast encryption and efficient instantiations. In: Roy, B. (ed.) ASIACRYPT 2005. LNCS, vol. 3788, pp. 100-120. Springer, Heidelberg (2005)

2. Boneh, D., Boyen, X., Goh, E.-J.: Hierarchical identity based encryption with constant size ciphertext. In: Cramer, R.J.F. (ed.) EUROCRYPT 2005. LNCS, vol. 3494, pp. 440-456. Springer, Heidelberg (2005)

3. Boneh, D., Franklin, M.: An efficient public key traitor tracing scheme. In: Wiener, M.J. (ed.) CRYPTO 1999. LNCS, vol. 1666, pp. 338-353. Springer, Heidelberg (1999)

4. Boneh, D., Gentry, C., Waters, B.: Collusion resistant broadcast encryption with short ciphertexts and private keys. In: Shoup, V. (ed.) CRYPTO 2005. LNCS, vol. 3621, pp. 258-275. Springer, Heidelberg (2005)

5. Boneh, D., Waters, B.: A fully collusion resistant broadcast, trace, and revoke system. In: Proceedings of the ACM Conference on Computer and Communications Security - CCS 2006, pp. 211-220. ACM Press, New York (2006)

6. Dodis, Y., Fazio, N.: Public key broadcast encryption for stateless receivers. In: Feigenbaum, J. (ed.) DRM 2002. LNCS, vol. 2696, pp. 61-80. Springer, Heidelberg (2003)

7. Dodis, Y., Fazio, N.: Public key broadcast encryption secure against adaptive chosen ciphertext attack. In: Desmedt, Y.G. (ed.) PKC 2003. LNCS, vol. 2567, pp. 100-115. Springer, Heidelberg (2002)

8. Fiat, A., Naor, M.: Broadcast encryption. In: Stinson, D.R. (ed.) CRYPTO 1993. LNCS, vol. 773, pp. 480-491. Springer, Heidelberg (1994)

9. Fujisaki, E., Okamoto, T.: Secure integration of asymmetric and symmetric encryption schemes. In: Wiener, M.J. (ed.) CRYPTO 1999. LNCS, vol. 1666, pp. 537-554. Springer, Heidelberg (1999) 
10. Galindo, D.: Boneh-Franklin identity based encryption revisited. In: Caires, L., Italiano, G.F., Monteiro, L., Palamidessi, C., Yung, M. (eds.) ICALP 2005. LNCS, vol. 3580, pp. 791-802. Springer, Heidelberg (2005)

11. Goodrich, M.T., Sun, J.Z., Tamassia, R.: Efficient Tree-Based Revocation in Groups of Low-State Devices. In: Franklin, M. (ed.) CRYPTO 2004. LNCS, vol. 3152, pp. 511-527. Springer, Heidelberg (2004)

12. Halevy, D., Shamir, A.: The LSD broadcast encryption scheme. In: Yung, M. (ed.) CRYPTO 2002. LNCS, vol. 2442, pp. 47-60. Springer, Heidelberg (2002)

13. Kurosawa, K., Desmedt, Y.: Optimum traitor tracing and asymmetric schemes. In: Nyberg, K. (ed.) EUROCRYPT 1998. LNCS, vol. 1403, pp. 145-157. Springer, Heidelberg (1998)

14. Kurosawa, K., Yoshida, T.: Linear code implies public-key traitor tracing. In: Naccache, D., Paillier, P. (eds.) PKC 2002. LNCS, vol. 2274, pp. 172-187. Springer, Heidelberg (2002)

15. Lee, J.W., Hwang, Y.H., Lee, P.J.: Efficient public key broadcast encryption using identifier of receivers. In: Chen, K., Deng, R., Lai, X., Zhou, J. (eds.) ISPEC 2006. LNCS, vol. 3903, pp. 153-164. Springer, Heidelberg (2006)

16. Naor, D., Naor, M., Lotspiech, J.: Revocation and tracing schemes for stateless receivers. In: Kilian, J. (ed.) CRYPTO 2001. LNCS, vol. 2139, pp. 41-62. Springer, Heidelberg (2001)

17. Naor, M., Pinkas, B.: Efficient trace and revoke schemes. In: Frankel, Y. (ed.) FC 2000. LNCS, vol. 1962, pp. 1-20. Springer, Heidelberg (2001)

18. Shamir, A.: How to share a secret. Communications of the ACM 22(11), 612-613 (1979)

19. Tzeng, W.-G., Tzeng, Z.-J.: A public-key traitor tracing scheme with revocation using dynamic shares. In: Kim, K.-c. (ed.) PKC 2001. LNCS, vol. 1992, pp. $207-$ 224. Springer, Heidelberg (2001)

20. Wang, P., Ning, P., Reeves, D.S.: Storage-efficient stateless group key revocation. In: Zhang, K., Zheng, Y. (eds.) ISC 2004. LNCS, vol. 3225, pp. 25-38. Springer, Heidelberg (2004)

21. Yoo, E.S., Jho, N.-S., Cheon, J.J., Kim, M.-H.: Efficient broadcast encryption using multiple interpolation methods. In: Park, C.-s., Chee, S. (eds.) ICISC 2004. LNCS, vol. 3506, pp. 87-103. Springer, Heidelberg (2005)

22. Yoshida, M., Fujiwara, T.: An efficient traitor tracing scheme for broadcast encryption. In: Proceedings of 2000 IEEE International Symposium on Information Theory, p. 463. IEEE Press, Los Alamitos (2000) 